\title{
Resistência ao cisalhamento da linha de cola em madeiras tropicais amazônicas
}

\author{
Shear bonding strength of some Amazonian woods
}

\author{
Thalles Santiago Pimentel ${ }^{1}$ (1), Peter Wimmer ${ }^{2}$ (1) , Helder Resende de Carvalho $^{2}$ (D), \\ Luciano Roitman² (1D, Cláudio Henrique Soares Del Menezzi ${ }^{1}$ (1) \\ 'Universidade de Brasília - UnB. Brasília, DF, Brasil \\ ${ }^{2}$ Laboratório de Produtos Florestais - LPF. Brasília, DF, Brasil
}

Como citar: Pimentel, T. S., Wimmer, P., Carvalho, H. R., Roitman, L., \& Del Menezzi, C. H. S. (2021). Resistência ao cisalhamento da linha de cola em madeiras tropicais amazônicas. Scientia Forestalis, 49(132), e3753. https://doi.org/10.18671/scifor.v49n132.19

\begin{abstract}
Resumo
As florestas nativas e plantadas são responsáveis por atender a demanda interna do país por madeira, que conta com um mercado consumidor cada vez mais exigente por qualidade e conhecimento da origem dessas madeiras, assim uma forma de diversificar e agregar valor para as madeiras nativas é através da fabricação de produtos engenheirados de madeira (PEM). Este trabalho teve como objetivo principal averiguar o desempenho da colagem dos corpos de prova. O material lenhoso foi coletado na Unidade de Manejo Florestal de área de concessão federal do Serviço Florestal Brasileiro, explorado pela empresa Madeflona na Floresta Nacional de Jacundá, estado de Rondônia. As espécies estudadas foram, Allantoma decandra (Jequitibá), Dipteryx odorata (Cumaru Ferro), Dipteryx magnifica (Cumaru Rosa), Erisma uncinatum (Cedrinho), Handroanthus incanus (Ipê), Martiodendron elatum (Tamarindo), Peltogyne lecointei (Roxinho) foram utilizados acetato de polivinila crosslink (PVAc) e poliuretano (PUR). A gramatura utilizada foi de $200 \mathrm{~g} / \mathrm{m}^{2}$ para ambos adesivos, conforme a orientação dos fabricantes. Os corpos de prova foram confeccionados conforme a norma ASTM D 905-08, nas dimensões de 44,4 mm x 50,8 mm x 19,0 mm, nos sentidos longitudinal, radial e tangencial, respectivamente. Para o ensaio de cisalhamento na linha de cola (CLC), foram confeccionados 60 corpos de prova para cada espécie, sendo 30 para cada adesivo. Para comparação, também foi realizado o cisalhamento paralelo às fibras na madeira sólida, seguindo a norma ASTM D 143-94, com a confecção de 12 corpos de prova por espécie. Avaliou-se a percentagem de falha na linha de cola/madeira, utilizando um gabarito quadriculado, subdividido em 100 quadrados menores. Também foi calculada a eficiência dos adesivos, através de uma relação do cisalhamento na linha de cola dividida pelo cisalhamento da madeira sólida. Para as espécies do estudo tem-se duas classificações quanto a densidade básica, madeiras de densidade alta e madeiras de densidade média. Os resultados do cisalhamento da madeira foram $D$. odorata $=16,23 \mathrm{MPa}, H$. incanus $=15,44 \mathrm{MPa}$, P. lecontei $=16,43 \mathrm{MPa}, D$. magnifica $=16,51 \mathrm{MPa}, \mathrm{M}$. elatum $=13,95 \mathrm{MPa}$, $A$. decandra $=10,81 \mathrm{MPa} \mathrm{e}$ E. uncinatum $=8,89 \mathrm{MPa}$. No ensaio de CLC utilizando o PVAc, $P$. lecointei apresentou melhor desempenho com média de 19,33 MPa, seguido do $D$. odorata com média de 17,18 MPa, porém não houve diferença com M. elatum (13,52 MPa), H. incanus (14,42 MPa), D. magnifica (14,31 MPa) e A. decandra (15,04MPa) e o que apresentou menor desempenho foi o E. uncinatum com média de 12,12 MPa. Para o ensaio de CLC utilizando o PUR, D. odorata (15,16 MPa), H. incanus (14,47 MPa), A. decandra (14,14 MPa) e D. magnifica $(13,68 \mathrm{MPa})$ não diferiram entre si, apresentando os melhores resultados. Por outro lado, as espécies $M$. elatum (12,56 MPa), P. lecointei (11,92 MPa) e E. uncinatum (11,79 MPa) obtiveram um resultado inferior e as suas médias não diferiram entre si. Para a falha na madeira obteve-se valores médios para o PVAc de $65 \%$ e para o PUR de 30\%. Para a eficiência das espécies que alcançaram um valor maior que $90 \%$ foram P. lecointei (PVAc), E. uncinatum (PVAc e PUR), M. elatum (PVAc e PUR), H. incanus (PVAc e PUR), D. odorata
\end{abstract}

Fonte de financiamento: Coordenação de Aperfeiçoamento de Pessoal de Nível Superior (CAPES).

Conflito de interesse: Nada a declarar

Autor correspondente: thallessantiagopimentel@hotmail.com

Recebido: 06 agosto 2021.

Aceito: 26 novembro 2021 .

Editor: Mauro Valdir Schumacher. 
(PVAc e PUR) e A. decandra (PVAc e PUR). Observou-se que a resistência na linha de cola para todas as espécies e a porcentagem de falha na madeira foi maior quando aplicado o adesivo PVAc em relação ao adesivo PUR, embora algumas espécies não apresentassem diferença estatística entre elas para o ensaio de CLC.

Palavras-chave: Colagem de madeiras; Madeiras nativas; Propriedades mecânicas.

\begin{abstract}
Native and planted forests are responsible for meeting the country's internal demand for wood, which has an increasingly demanding consumer market for quality and knowledge of the origin of these woods; therefore one way to diversify and add value to native wood is through the manufacture of engineered wood products (PEM). The aim of this work was to investigate the performance of bonding specimens. The woody material was collected in the Forest Management Unit of a federal concession area of the Brazilian Forest Service, explored by the company Madeflona in the Jacundá National Forest, state of Rondônia. The studied species were Allantoma decandra (Jequitiba), Dipteryx odorata (Cumaru Ferro), Dipteryx magnifica (Cumaru Rosa), Erisma uncinatum (Cedrinho), Handroanthus incanus (Ipe), Martiodendron elatum (Tamarindo), Peltogyne lecointei (Roxinho) and the methods used were crosslink polyvinyl acetate (PVAc) and polyurethane (PUR). Gluing was done with a weight of $200 \mathrm{~g} / \mathrm{m}^{2}$ for both adhesives, as instructed by the manufacturers. The specimens were made according to the ASTM D 905-08 standard, with dimensions of 44,4 $\mathrm{mm} \times 50,8 \mathrm{~mm} \times 19,0 \mathrm{~mm}$ in the longitudinal, radial and tangential directions, respectively. For the glue line shear test (GLS), 60 specimens were made for each species, 30 for each adhesive. For comparison, the shear parallel to the fibers in solid wood was also performed, following the ASTM D 143-94 standard, with the preparation of 12 specimens per species. The percentage of failure in the glue/wood line was evaluated using a checkered template, subdivided into 100 smaller squares. The efficiency of the adhesives was calculated through a ratio of the shear in the glue line divided by the shear of solid wood. For the species in the study we used two classifications regarding basic density, high density wood and medium density wood. The results obtained for wood shear were: $D$. odorata $=16.23 \mathrm{MPa}, H$. incanus $=15.44 \mathrm{MPa}$, $P$. lecontei $=16.43 \mathrm{MPa}, D$. magnifica $=16.51 \mathrm{MPa}, M$. elatum $=13.95 \mathrm{MPa}, A$. decandra $=10.81 \mathrm{MPa}$ and $E$. uncinatum $=8.89 \mathrm{MPa}$, which were the mean values for the GLS assay using PVAc. TheTukey test revealed that $P$. lecointei showed better performance with an average of $19.33 \mathrm{MPa}$, followed by $D$. odorata with an average of $17.18 \mathrm{MPa}$; while there was no difference between M. elatum (13.52 MPa), H. incanus (14.42 MPa), D. magnifica (14.31 MPa) and $A$. decandra (15.04 MPa). The one with the lowest performance was $E$. uncinatum with an average of 12.12 MPa. The mean values for the GLS assay using PUR, indicated that D. odorata (15.16 MPa), H. incanus (14.47 MPa), A. decandra (14.14 MPa) and D. magnifica (13.68 MPa) did not differ and were the best results. On the other hand, the species M. elatum (12.56 MPa), P. lecointei (11.92 MPa) and E. uncinatum (11.79 MPa) yielded a lower result and their means did not differ significantly from each other. For wood failure mean values for PVAc of $65 \%$ and PUR of $30 \%$ were obtained. For efficiency, species that reached a value greater than $90 \%$ were $P$. lecointei (PVAc), $E$. uncinatum (PVAc and PUR), M. elatum (PVAc and PUR), H. incanus (PVAc and PUR), D. odorata (PVAc and PUR) and A. decandra (PVAc and PUR). It was observed that the glue line strength for all species and the percentage of wood failure was higher when the PVAc adhesive was applied, as compared to the PUR adhesive, although some species did not show any statistical difference between them in the GLS test.
\end{abstract}

Keywords: Wood bonding; Native woods; Mechanical properties.

\title{
INTRODUÇÃO
}

As florestas nativas e plantadas são responsáveis por atender a demanda interna do país por madeira. De acordo com Souza et al. (2020), o mercado consumidor está cada vez mais exigente com a qualidade e a origem dessas madeiras, dessa forma, o conhecimento das propriedades tecnológicas e sua aplicabilidade são necessárias para diversificar o seu uso. Comutativamente, a exploração dos recursos naturais deve ser realizada por um Plano de Manejo Florestal Sustentável regulamentado por um órgão fiscalizador competente, que visa garantir a contínua produção e proteção da área explorada (Stragliotto et al., 2020).

Uma forma de diversificar e agregar valor para as madeiras nativas é através da fabricação de produtos engenheirados da madeira (PEM), que consistem num amplo conjunto de produtos processados industrialmente que utilizam peças de madeira unidas por adesivo, a fim de otimizar seu uso na construção, movelaria, etc. De acordo com Segundinho et al. (2017), os setores que utilizam madeira maciça buscaram novas alternativas para um aproveitamento mais racional e que pudessem diminuir suas limitações de uso. Por 
conseguinte, o desenvolvimento de adesivos sintéticos tornou possível a união de peças de madeira para criar membros estruturais.

Antes de realizar a manufatura de PEM como, por exemplo, madeira laminada colada (MLC) e madeira laminada colada cruzada (CLT), painel colado lateralmente e colagem de topo, deve-se testar a eficiência do adesivo através de ensaios de cisalhamento na linha de cola (CLC), tração normal à face colada, delaminação, entre outros. Diante disso, o adesivo é o responsável por transferir tensões de uma peça para a outra, na qual o fenômeno físico-químico que explica o comportamento do adesivo de aderir a madeira é a adesão, o qual prediz um mecanismo de interação entre superfícies sólidas, com capacidade de manter unidos outros materiais em sua superfície (Iwakiri et al., 2000).

Para uma colagem de sucesso é necessário que o adesivo tenha um desempenho superior ao da madeira, ou seja, as tensões devem gerar um rompimento na madeira e não na linha de cola. A alta porcentagem de falha na madeira, associada ao elevado valor da tensão de cisalhamento, indica uma colagem bem sucedida, todavia se a porcentagem de falha se encontrar na linha de cola, isso caracteriza uma baixa adesão e/ou baixa coesão na interface com a madeira, devido a fatores externos relacionados à interação da madeira e o adesivo (Oliveira \& Seraphim, 2015).

Para a colagem, as condições físicas e químicas da superfície são importantes, neste sentido com viscosidade adequada dos adesivos para umedecer e espalhar nas superfícies, associado à aplicação de pressão para melhor consolidar as juntas de madeira coladas, são requisitos indispensáveis para uma colagem de qualidade (Santiago et al., 2018). O adesivo é um componente importante na produção de produtos à base de madeira e seus derivados, que pode representar até 50\% do custo total do painel (Varanda et al., 2018).

Partindo dessas informações, o presente trabalho objetivou averiguar o desempenho da colagem de sete espécies de madeiras tropicais amazônicas utilizando adesivos de acetato de polivinila crosslink (PVAc) e poliuretano (PUR), buscando assim, a diversificação de uso para madeiras nativas e suas possíveis aplicações para o desenvolvimento de produtos engenheirados da madeira.

\section{MATERIAL E MÉTODOS}

\section{Material lenhoso avaliado}

O material lenhoso foi coletado na Unidade de Manejo Florestal da área de concessão federal do Serviço Florestal Brasileiro explorado pela empresa Madeflona na Floresta Nacional de Jacundá, estado de Rondônia, Brasil. Foram coletados entre três e cinco indivíduos de cada uma das sete espécies (Tabela 1), buscando contemplar a variação interespecífica. O trabalho foi realizado na Área de Engenharia e Física da Madeira do Laboratório de Produtos Florestais (LPF) do Serviço Florestal Brasileiro (SFB).

Tabela 1. Relação das sete espécies contempladas neste estudo, coletadas na Floresta Nacional do Jacundá.

\begin{tabular}{ccc}
\hline Nome Científico & Família Botânica & Nome Comum \\
\hline Allantoma decandra & Lecythidaceae & Jequitibá \\
Dipteryx magnifica & Fabaceae & Cumaru Rosa \\
Dipteryx odorata & Fabaceae & Cumaru Ferro \\
Erisma uncinatum & Vochysiaceae & Cedrinho \\
Handroanthus incanus & Bignoniaceae & Ipê Amarelo \\
Martiodendron elatum & Fabaceae & Tamarindo \\
Peltogyne lecointei & Fabaceae & Roxinho \\
\hline
\end{tabular}

Após desdobro inicial na serraria da concessionária em Rondônia, as madeiras foram transportadas até as dependências do Laboratório de Produtos Florestais (LPF) em Brasília, onde foram processadas dando origem aos corpos de prova (CPs) utilizados no estudo. 


\section{Determinação da densidade básica da madeira}

Realizou-se a determinação da densidade básica da madeira das espécies estudadas, conforme a norma da American Society for Testing and Materials, ASTM D-2395 (American Society for Testing and Materials, 2014). As dimensões dos corpos de prova utilizados foram de $5,0 \mathrm{~cm} \times 3,0 \mathrm{~cm} \times 2,0 \mathrm{~cm}$, nos sentidos longitudinal, radial e tangencial, respectivamente. Inicialmente as amostras foram submersas em água, até saturação completa para determinação do volume, que foi aferido pelo método de imersão e, após submetidas a secagem em estufa a $103{ }^{\circ} \mathrm{C}$, até peso constante, para obtenção da massa seca, que foi registrada com auxílio de uma balança digital de duas casas decimais $(0,01 \mathrm{~g})$. De posse destes valores calculou-se a densidade básica, conforme a Equação 1.

$d b=\frac{m s}{v s}$

Em que:

$\mathrm{db}=$ densidade básica, em $\mathrm{g} / \mathrm{cm}^{3}$;

$\mathrm{ms}=$ massa seca, em g;

vs $=$ volume saturado, $\mathrm{em}_{\mathrm{cm}}^{3}$.

\section{Confecção dos corpos de prova para o teste de CLC}

Os tarugos de dimensões $150,0 \mathrm{~cm} \times 5,0 \mathrm{~cm} \times 5,0 \mathrm{~cm}$, nos sentidos longitudinal, radial e tangencial, respectivamente, foram aplainados antes da colagem, respeitando o intervalo máximo de 24 horas entre o aplainamento e a aplicação dos adesivos, cujas propriedades estão descritas na Tabela 2. A gramatura utilizada, para ambos os adesivos, foi de $200 \mathrm{~g} / \mathrm{m}^{2}$, conforme orientação dos fabricantes. Foram utilizados acetato de polivinila crosslink (PVAc) e poliuretano (PUR), pois a utilização desses adesivos já está difundida nos mercados de laminação, movelaria e painéis sarrafeados, sendo o PVAc usado para ambientes internos e o PUR para ambientes externos.

Tabela 2. Características dos adesivos utilizados para a colagem das espécies do estudo.

\begin{tabular}{lccccc}
\hline \multicolumn{1}{c}{ Adesivo } & Cor & $\begin{array}{c}\text { Teor de } \\
\text { sólidos (\%) }\end{array}$ & $\begin{array}{c}\text { Viscosidade } \\
\text { (cPs) }\end{array}$ & pH & $\begin{array}{c}\text { Densidade } \\
\left(\mathbf{g} / \mathbf{c m}^{\mathbf{3}}\right)\end{array}$ \\
\hline PVAc da marca Titebond II & $\begin{array}{c}\text { Creme } \\
\text { amarelada }\end{array}$ & 48 & 4000 & 3 & 1,09 \\
Premium & Mel & $*$ & 8000 & $*$ & 1,13 \\
PUR da marca Kleiberit & * &
\end{tabular}

PVAc (acetato de polivinila crosslink); PUR (poliuretano); * não informado pelo fabricante

A aplicação dos adesivos nos tarugos ocorreu sobre uma balança de precisão de duas casas decimais e com auxílio de uma espátula para espalhar o adesivo na face colada. Em seguida foram prensados numa prensa hidráulica da marca Indumec utilizando a pressão de $12 \mathrm{kgf} / \mathrm{cm}^{2}(1,17 \mathrm{MPa})$ para as espécies com madeiras de densidades médias (Allantoma decandra e Erisma uncinatum) e $15 \mathrm{kgf} / \mathrm{cm}^{2}(1,47 \mathrm{MPa})$ para as espécies com madeiras de densidade alta (Dipteryx magnifica, Dipteryx odorata, Handroanthus incanus, Martiodendron elatum e Peltogyne lecointei).

Após os tarugos serem colados e submetidos à prensa hidráulica, eles foram condicionados na sala de climatização em temperatura de $22^{\circ} \mathrm{C}$ e umidade relativa do ar de $63 \%$, por uma semana. Em seguida, foram levados para carpintaria para dar origem aos corpos de prova (CPs) conforme a norma ASTM D 905-08 (American Society for Testing and Materials, 2008) (Figura 1), nas dimensões de 44,4 mm x 50,8 $\mathrm{mm} \times 19,0 \mathrm{~mm}$, nos sentidos longitudinal, radial e tangencial, respectivamente. 


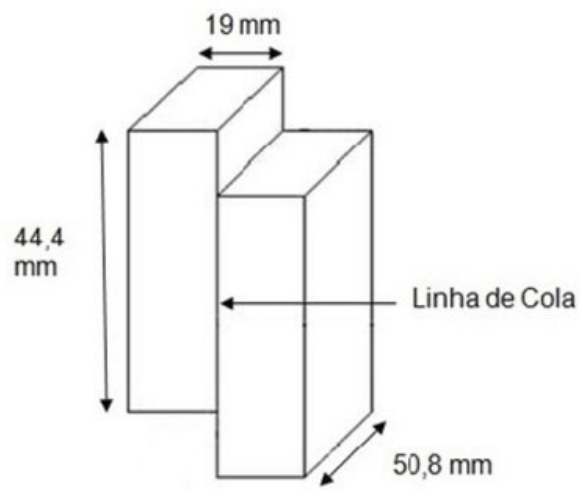

Figura 1. Dimensões do corpo de prova para teste de CLC, conforme norma ASTM D 905-08 (American Society for Testing and Materials, 2008).

\section{Ensaio de cisalhamento, falha na madeira e eficiência}

Para o ensaio de CLC, foram confeccionados 60 corpos de prova para cada espécie, sendo 30 para cada adesivo. Logo após a confecção, os CPs foram testados numa máquina Universal de Ensaios da marca Martins Campelo, seguindo a norma ASTM D905-08, com o registro da carga e tensão máxima de cisalhamento a uma velocidade de $5 \mathrm{~mm} / \mathrm{min}$.

Para comparação, também foi realizado o cisalhamento paralelo às fibras na madeira sólida, seguindo a norma ASTM D 143-94 (American Society for Testing and Materials, 1994) (Figura 2), com a confecção de 12 corpos de prova por espécie. Antes da realização dos testes, as amostras foram mensuradas com o auxílio de um paquímetro digital, de acordo com os planos direcionais da madeira quantificando altura, largura e espessura.

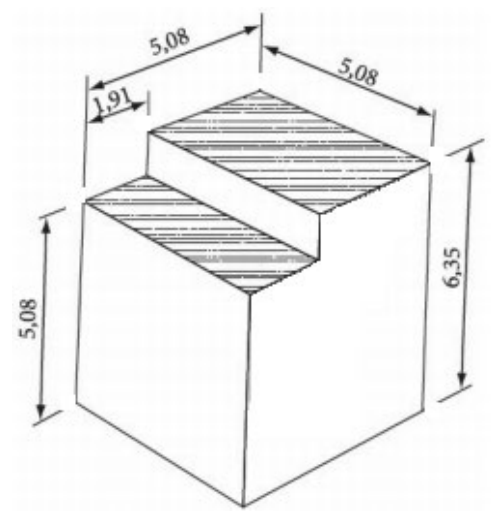

Figura 2. Dimensões dos corpos de prova para o teste de cisalhamento paralelo às fibras na madeira sólida.

Também avaliou-se a percentagem de falha na linha de cola/madeira, utilizando um gabarito quadriculado, subdividido em 100 quadrados menores.

A eficiência foi calculada com base na Equação 2, na qual utiliza-se os dados do cisalhamento de cada adesivo em relação ao cisalhamento da madeira das espécies.

$$
E(\%)=\frac{f_{g v, 0}}{f_{v, 0}} \times 100
$$

Em que:

$E(\%)=$ eficiência em porcentagem; 
$f_{g v, 0}=$ resistência ao cisalhamentona linha de cola, $M P a ;$

$f_{v, 0}=$ resistência ao cisalhamentolongitudional da madeira sólida, MPa .

\section{Análise estatística}

Para análise estatística foi utilizado o software R Studio, na qual foram aferidos os outliers através de uma plotagem boxplot antes de realizar as análises. Foi aplicado um teste de Shapiro-Wilk para verificar a normalidade dos dados. O delineamento estatístico adotado foi o inteiramente casualizado, sendo sete tratamentos para a avaliação da resistência na linha de cola e falha na madeira; submetendo-os à análise de variância comparando os adesivos dentro de cada espécie e entre os adesivos empregados.

Para avaliar a diferença entre os tratamentos dos dados que seguiram uma distribuição normal, foi aplicado um teste de Tukey, e para os dados que não seguiram uma distribuição normal foi aplicado um teste não paramétrico de Wilcoxon, ambos os testes foram comparados a uma significância de $5 \%$.

O teste não paramétrico de Wilcoxon realiza análises pareando os dados de uma mesma variável dependente, utilizando as medianas como medida de tendência central de referência e usa a amplitude interquartil como medida de dispersão. Desta forma, o teste comparou aos pares as espécies, realizando no total 21 interações entre elas, mostrando qual espécie diferia da outra a uma significância de $5 \%$.

\section{RESULTADOS E DISCUSSÃO}

\section{Densidade básica e cisalhamento da madeira sólida}

Para as espécies do estudo têm-se duas classificações quanto a densidade básica, madeiras de densidade alta ( $D$. odorata, $H$. incanus, $P$. lecointei, $D$. magnifica e $M$. elatum) e madeiras de densidade média (A. decandra e E. uncinatum); o cisalhamento da madeira sólida tem-se valores altos, com exceção do $E$. uncinatum $(8,89 \mathrm{MPa})$ que em comparação com A. decandra (10,81 MPa) apresentou um valor inferior (Tabela 3).

Tabela 3. Valores médios de densidade básica e cisalhamento da madeira sólida para as espécies tropicais amazônicas.

\begin{tabular}{ccc}
\hline Nome Científico & Densidade Básica $\mathbf{( g / \mathbf { c m } ^ { 3 } )}$ & $\begin{array}{c}\text { Cisalhamento da } \\
\text { Madeira Sólida (MPa) }\end{array}$ \\
D. odorata & 0,92 & 16,23 \\
H. incanus & 0,89 & 15,44 \\
P. lecointei & 0,86 & 16,43 \\
D. magnifica & 0,82 & 16,51 \\
M. elatum & 0,85 & 13,95 \\
A. decandra & 0,57 & 10,81 \\
E. uncinatum & 0,55 & 8,89 \\
\hline
\end{tabular}

A resistência ao cisalhamento da madeira é diretamente proporcional à sua densidade, ou seja, valores maiores de densidade básica obtém-se resultados superiores de cisalhamento da madeira sólida. 


\section{Cisalhamento na linha de cola}

O teste de normalidade de Shapiro-Wilk para os dados de CLC indicaram uma distribuição normal. A análise de variância (ANOVA), constatou que há diferença a uma significância de $5 \%$, havendo diferenças entre as espécies estudadas.

Os valores médios para o ensaio de CLC utilizando o PVAC (Figura 3), na qual o teste de Tukey constatou que o $P$. lecointei apresentou melhor desempenho com média de 19,33 MPa, seguido do $D$. odorata com média de $17,18 \mathrm{MPa}$, porém não houve diferença com $M$. elatum (13,52 MPa), H. incanus (14,42 MPa), D. magnifica (14,31 MPa) e A. decandra (15,04MPa) e o que apresentou menor desempenho foi o E. uncinatum com média de 12,12 MPa.

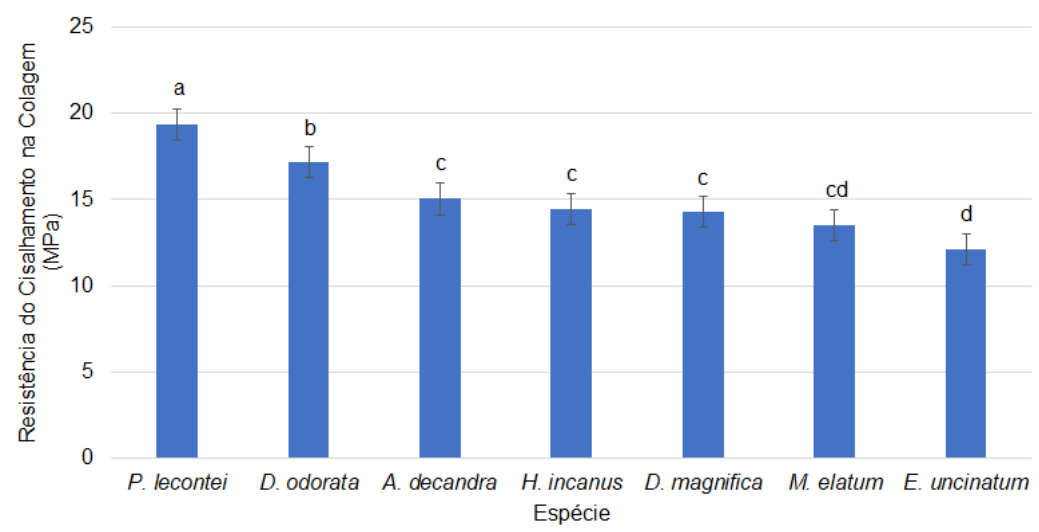

Figura 3. Valor médio do CLC com o uso de PVAc para espécies tropicais amazônicas. Nota: médias seguidas de mesma letra não diferem estatisticamente entre si pelo teste de Tukey a 5\% de significância.

Para a variável PUR a plotagem do boxplot, identificou dados fora do intervalo para as espécies $P$. lecontei, E. uncinatum, $D$. odorata, $D$. magnifica e $A$. decandra. Após a retirada dos outliers foi realizada uma análise de variância, na qual foi constatado diferença a uma significância de $5 \%$.

Os valores médios para o ensaio de CLC utilizando o PUR (Figura 4), em que o teste de Tukey indicou que $D$. odorata (15,16 MPa), H. incanus (14,47 MPa), A. decandra (14,14 MPa) e D. magnifica $(13,68 \mathrm{MPa})$ não diferiram entre si, apresentando os melhores resultados. Por outro lado, as espécies M. elatum (12,56 MPa), P. lecointei (11,92 MPa) e E. uncinatum (11,79 $\mathrm{MPa}$ ) obtiveram um resultado inferior e as suas médias não diferiram entre si.

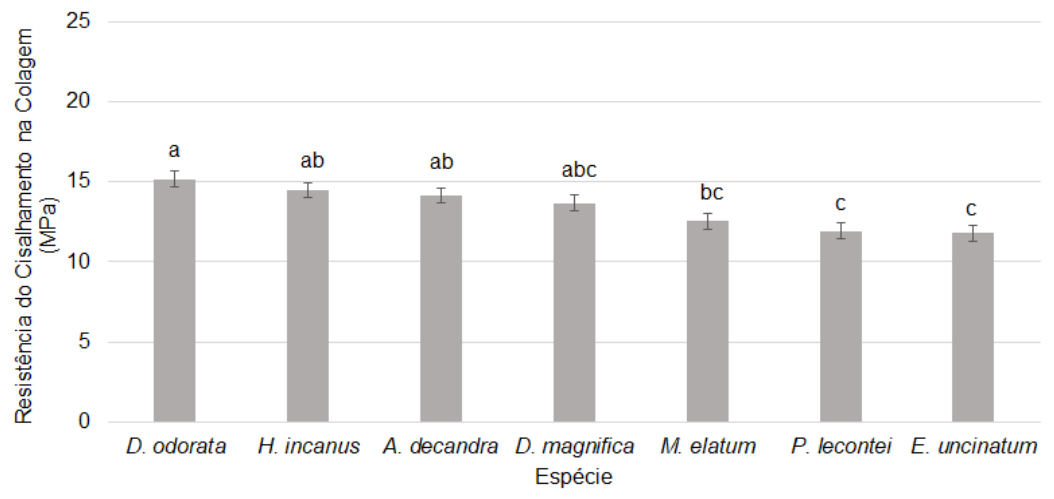

Figura 4. Valor médio do CLC com o uso de PUR para as espécies tropicais amazônicas. Nota: médias seguidas de mesma letra, não diferem estatisticamente entre si pelo teste de Tukeya 5\% de significância.

As propriedades anatômicas da madeira possuem uma significativa influência na colagem de madeiras, como a variabilidade na densidade e porosidade que ocorre devido aos lenhos iniciais e tardios (He et al., 2019). Contudo, pode-se inferir que a densidade é o 
principal motivo para uma colagem de sucesso considerando as variáveis da madeira, assim, correlacionando madeiras de densidade alta com uma baixa penetração do adesivo, devido às paredes celulares mais espessas, lúmens e pontuações reduzidos.

Assim como as propriedades anatômicas desempenham um papel importante para a colagem, têm-se as propriedades químicas da madeira, na qual interfere diretamente na qualidade das faces coladas. De acordo com Roffael (2016), os extrativos da madeira por serem solventes orgânicos acabam interagindo com o adesivo, podendo enfraquecer as ligações de hidrogênio e dipolo-dipolo.

A Tabela 4 apresenta os valores determinados para o cisalhamento na linha de cola. Assim, é possível observar que as espécies $H$. incanus, D. magnifica, M. elatum e $E$. uncinatum não diferem entre a utilização de PVAc ou PUR.

Tabela 4. Resistência do cisalhamento na linha de cola em espécies tropicais amazônicas.

\begin{tabular}{ccccc}
\hline \multirow{2}{*}{ Nome Científico } & \multicolumn{4}{c}{ Cisalhamento na linha de cola (MPa) } \\
\cline { 2 - 5 } & PVAc & CV (\%) & PUR & CV (\%) \\
\hline D. odorata & $17,19^{* *}$ & 15,02 & $15,16^{* *}$ & 18,33 \\
H. incanus & $14,47^{\text {ns }}$ & 23,82 & $14,43^{\text {ns }}$ & 21,02 \\
P. lecointei & $19,33^{* *}$ & 9,86 & $11,93 * *$ & 22,00 \\
D. magnifica & $14,31^{\text {ns }}$ & 25,92 & $13,68^{\text {ns }}$ & 13,10 \\
M. elatum & $13,52^{\text {ns }}$ & 22,82 & $12,56^{\text {ns }}$ & 26,14 \\
A. decandra & $15,04^{*}$ & 9,77 & $14,14 *$ & 9,53 \\
E. uncinatum & $12,12^{\text {ns }}$ & 10,42 & $11,80^{\text {ns }}$ & 15,43 \\
\hline
\end{tabular}

Nota: *valores médios na coluna para o cisalhamento na linha de cola, são diferentes a significância de 5\%; ** são significativamente diferentes a 1\%; ns não significativo a 5\%; CV (\%) coeficiente de variação em porcentagem.

A pressão aplicada para a consolidação da junta colada variou para as espécies de densidade média e alta, e isso pode ter contribuído para o desempenho do teste de CLC, cuja as espécies de densidade maior obtiveram um desempenho superior às espécies de densidade média. Santiago et al. (2018), relatam que a elevada resistência ao cisalhamento das juntas coladas com madeiras de densidade alta é atribuída às paredes celulares espessas capazes de suportar maiores tensões. Portanto, a maior pressão aplicada para as espécies de alta densidade ajudou a mobilizar o adesivo dentro da estrutura anatômica das madeiras, proporcionando uma maior resistência.

Para avaliar quais dos adesivos produzia a maior resistência na linha de cola foi realizado uma análise de variância, na qual foi constatado que há diferenças entre os adesivos. Os valores médios para a resistência na linha de cola com PVAc e PUR foram 15,13 MPa e 13,39 $\mathrm{MPa}$, respectivamente (Figura 5).

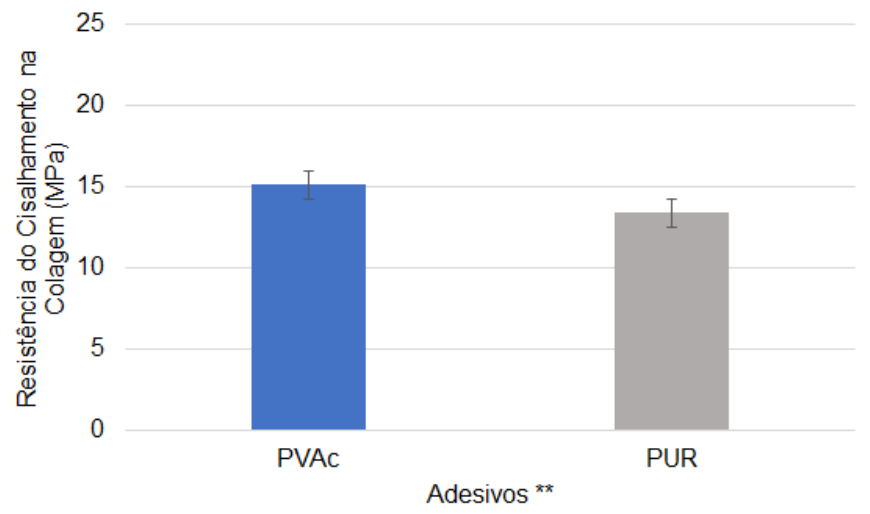

Figura 5. Valores médios de CLC em CPs colados com PVAc e PUR de espécies tropicais amazônicas. Nota: ** são significativamente diferentes a uma significância de $1 \%$. 


\section{Porcentagem de falha na madeira}

Avaliando a porcentagem de falha na madeira utilizando a densidade como parâmetro, ambos adesivos obtiveram os melhores resultados para as madeiras de média densidade Allantoma decandra e Erisma uncinatum (Tabela 5). Iwakiri (2005) explana a influência da mobilidade do adesivo nas propriedades anatômicas para a falha na madeira, em que é influenciada pela constituição dos anéis de crescimento, densidade, presença de lenho juvenil e disposição da grã.

Tabela 5. Valores da média de porcentagem de falha na madeira e coeficiente de variação para os adesivos PVAc e PUR em espécies tropicais amazônicas.

\begin{tabular}{ccccc}
\hline \multirow{2}{*}{ Espécie } & \multicolumn{4}{c}{ Falha na madeira (\%) } \\
\cline { 2 - 5 } & PVAc & CV (\%) & PUR & CV (\%) \\
\hline E. uncinatum & 97 & 3,68 & 84 & 16,27 \\
A. decandra & 72 & 11,00 & 56 & 52,10 \\
M. elatum & 81 & 13,11 & 32 & 75,80 \\
D. odorata & 70 & 41,42 & 15 & 55,63 \\
D. magnifica & 51 & 74,23 & 12 & 81,37 \\
P. lecointei & 44 & 63,78 & 8 & 45,62 \\
H. incanus & 40 & 76,48 & 2 & 137,16 \\
Média & 65 & & 30 & \\
\hline
\end{tabular}

O teste de normalidade de Shapiro-Wilk foi aplicado para os dados de falha na madeira, as duas variáveis apresentaram um p-valor menor que a significância de 5\%, desta maneira a hipótese alternativa foi aceita, ou seja, os dados não seguem uma distribuição normal e foi realizado um teste não paramétrico de Wilcoxon para averiguar as diferenças entre as espécies.

Para a mensuração da falha na madeira, pode-se atribuir a falta de normalidade dos dados por ser um teste em que a subjetividade do avaliador é de grande relevância, pois existe uma dificuldade de visualizar se a falha está na madeira ou na linha de cola. Este fenômeno é discutido por Lopes et al. (2013), na qual observaram uma alta variabilidade dos dados para a falha na madeira, atribuindo à preparação da linha de cola, à baixa ligação mecânica e química entre o adesivo e madeira e a subjetividade do avaliador na análise.

Antes de realizar o teste de Wilcoxon foram aferidos os outliers através de uma plotagem boxplot para os adesivos PVAc e PUR, assim os outliers foram retirados do banco de dados.

Para a porcentagem de falha na madeira com o uso do adesivo de PVAc (Figura 6), o teste de Wilcoxon demonstrou que a mediana de $E$. uncinatum apresentou o melhor desempenho com o valor de $95 \%$ de falha na madeira, apresentando uma diferença significativa com as demais espécies. As medianas das espécies M. elatum (85\%), D. odorata (85\%) e A. decandra (85\%) não apresentaram diferença significativa entre si. E por fim, as espécies de $P$. lecointei $(52,5 \%), H$. incanus (25\%) e $D$. magnifica $(75 \%)$ não obtiveram diferença estatística a um nível de $5 \%$ de significância entre si, que por sua vez apresentaram um desempenho inferior e significativo às outras 4 espécies.

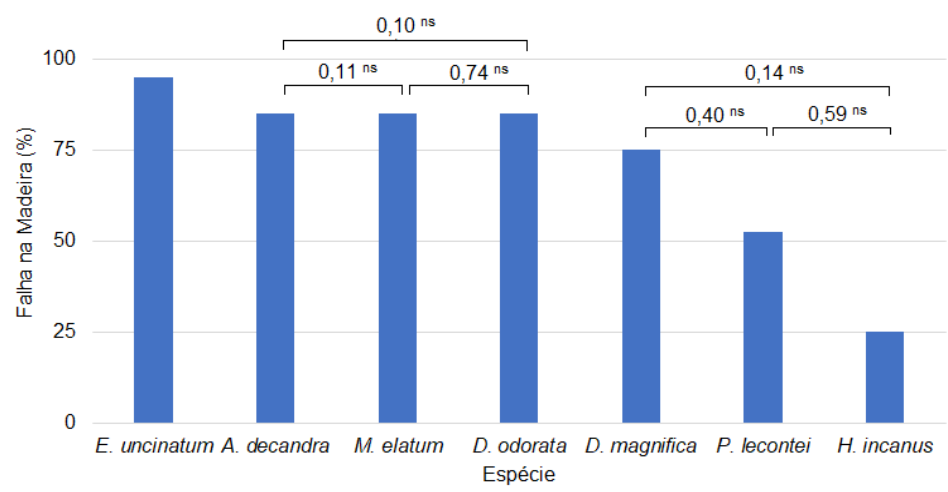

Figura 6. Mediana da porcentagem de falha na madeira com o uso de PVAc para as espécies tropicais amazônicas e respectivo resultado do teste de Wilcoxon, a 5\% de significância. Nota: p-valor não significativo (ns). 
Para a porcentagem de falha na madeira sob o uso do adesivo de PUR (Figura 7), o teste de Wilcoxon demonstrou que o $E$. uncinatum obteve melhor desempenho com a mediana de $90 \%$, seguido do $A$. decandra com mediana de $60 \%$ e $M$. elatum com mediana de $22,5 \%$. As únicas interações que não apresentaram diferenças foram entre $P$. lecointei $(10 \%)$ e D. magnifica (10\%) e para a interação de $D$. odorata (15\%) e D. magnifica, desta forma essas 4 espécies apresentam o pior desempenho na falha na madeira para a variável PUR.

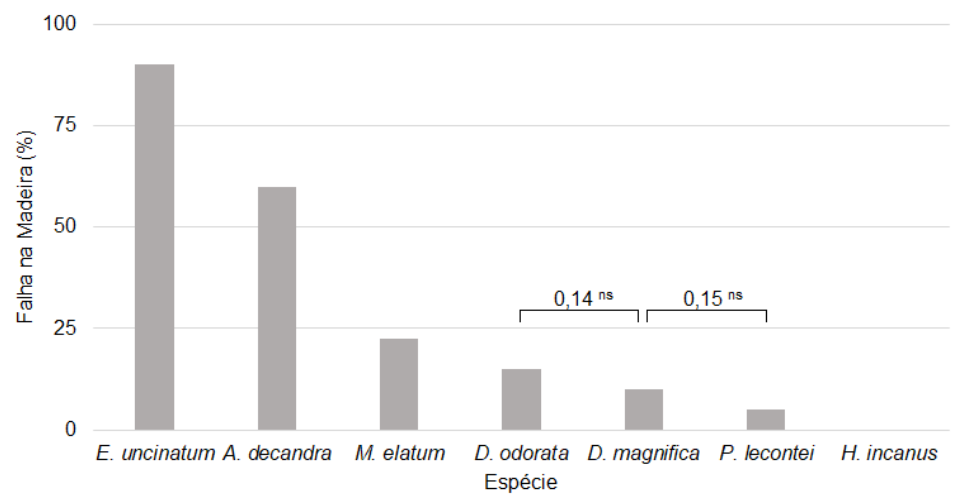

Figura 7. Mediana da porcentagem de falha na madeira com o uso de PUR para espécies tropicais amazônicas e respectivo resultado do teste de Wilcoxon, a 5\% de significância.

A porcentagem de falha na madeira, para as espécies do estudo, foi maior quando aplicado o adesivo PVAc (média de 65\% de falha) em relação ao adesivo PUR (média de 30\% de falha). Assim, pode-se inferir que a interação do adesivo PUR com as madeiras de alta densidade ( $D$. odorata, $D$. magnifica, $H$. incanus, $P$. lecointei e $M$. elatum) foi inferior devido à espessura das paredes celulares serem maiores e isso acaba prejudicando o intertravamento mecânico do adesivo à estrutura lenhosa. Para os autores Faria et al. (2020) a interação do adesivo com a madeira é melhor quantificada quando observada a porcentagem de falha na madeira, na qual maiores porcentagens de falha na madeira indicam que as ligações adesivas são maiores que a madeira, indicando um bom intertravamento mecânico.

\section{Eficiência}

O objetivo de quantificar a eficiência é avaliar o teste do adesivo em relação à madeira sólida, na qual o desejável é uma relação maior que 100\% (Figura 8).

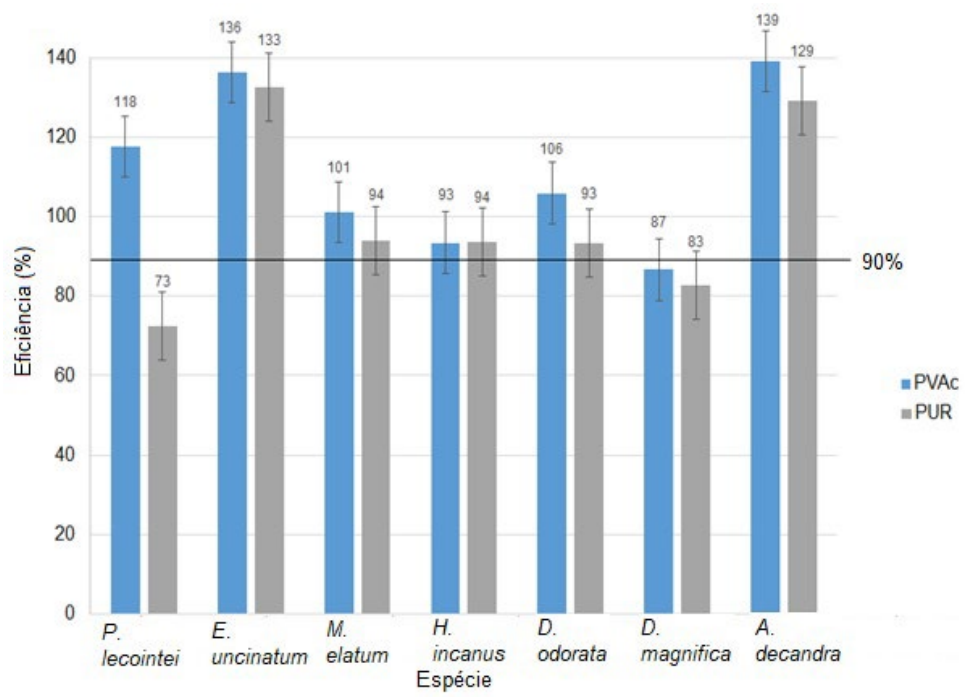

Figura 8. Valores médios de CLC divididos pelos valores médios de Cisalhamento da Madeira Sólida, para obtenção da eficiência em porcentagem, em espécies tropicais amazônicas. 
Uma eficiência maior que 90\%, indica uma maior confiança para a confecção de MLC, já que o principal teste para esse produto engenheirado é o CLC. Assim, das 14 possibilidades avaliando espécie $x$ adesivo, as espécies $P$. lecointei (PVAC), E. uncinatum (PVAc e PUR), M. elatum (PVAc e PUR), H. incanus (PVAc e PUR), D. odorata (PVAc e PUR) e A. decandra (PVAc e PUR) podem seguir para a próxima etapa de confecção de MLC.

No geral, pode-se afirmar que o PVAc teve uma eficiência melhor do que o PUR. Parte desse resultado pode ser explicado pelo fato do PVAc possuir uma viscosidade de $4000 \mathrm{cPs}$ e o PUR 8000 cPs. Bianche et al. (2017), afirmam que adesivos de baixa viscosidade tem um melhor espalhamento sobre a face da madeira, auxiliando a maior penetração do adesivo e sua absorção pela madeira.

Mendoza et al. (2017), explicam que para colagem na madeira são desejados valores médios de viscosidade, porquanto adesivos com viscosidade alta tem a tendência de se distribuir de forma irregular na madeira, causando uma linha de cola espessa, por causa da dificuldade de penetração do adesivo na madeira. Assim, para que ocorra essa ligação forte com a madeira, o adesivo deve alcançar a camada de madeira intacta abaixo da superfície, penetrando e preenchendo os lúmens, pontuação das células e as fendas da parede celular (Albuquerque \& Latorraca, 2000; Biazzon et al., 2019; Jakes et al., 2007).

A eficiência do PVAc em relação ao PUR já foi comprovada em outros trabalhos. Segundinho et al. (2017), testaram os adesivos ureia-formaldeído (UF), poliacetato de vinila (PVA), resorcinol-formaldeído (RF), melamina-ureia-formaldeído (MUF), poliuretano à base de óleo de mamona (PUR) e tanino-formaldeído (TF) com a madeira de Eucalyptus cloeziana (densidade básica de $0,626 \mathrm{~g} / \mathrm{cm}^{3}$ ) com e sem preservante de Arseniato de Cobre Cromatado (CCA) e constatou que a madeira sólida tratada teve uma resistência de 10,32 MPa e os adesivos PVA e PUR obtiveram um CLC de 12,85 MPa e 8,29 MPa, respectivamente. Dessa forma, o autor não calculou a eficiência no trabalho, mas utilizando os valores de cisalhamento da madeira sólida disponível no artigo, constata-se que a eficiência para o PVA foi de $124 \%$ e o PUR de $80 \%$, confirmando assim a eficiência do PVAc em relação ao PUR.

Os autores Santos \& Del Menezzi (2010), avaliaram Allantoma lineata e Simarouba amara (madeiras tropicais) fixadas com resorcinol-formaldeído nas gramaturas de 200, 300 e $400 \mathrm{~g} / \mathrm{cm}^{2}$ e obtiveram eficiência maior que $100 \%$ para a $A$. lineata nas gramaturas 300 e $400 \mathrm{~g} / \mathrm{cm}^{2}$.

O trabalho desenvolvido por Nascimento et al. (2013), avaliaram a resistência na linha de cola para espécies comerciais (Plathymenia reticulata Benth., Dinizia excelsa Ducke, Cedrela fissilis Vell., Cedrelinga catenaeformis Ducke, Ocotea rubra Mez, Pinus sp., Enterolobium maximum Ducke, Amburana cearensis (Fr. All.) A. C. Smith, Piptadenia suaveolens Miq., Bagassa guianensis Aubl., Apuleia leiocarpa (Vog.) Macbr., Hymenaea courbaril L., Dipteryx odorata (Aubl.) Willd. e Manilkara sp.), na qual classificaram em três níveis as densidades, onde classe 1:

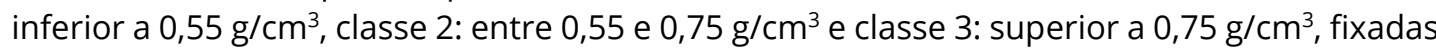
com os adesivos PVAc e ureia-formaldeído (UF). Os autores encontraram valores de eficiência para o PVAc maior que $100 \%$ para todas as espécies, indicando que a linha de cola foi mais resistente, por outro lado o uso de UF não foi maior que 100\% em todas as espécies.

Por fim, o uso de PVAc para a colagem é uma boa alternativa, se for para aplicações internas. Ferreira et al. (2012), mencionam que o PVAc pode se tornar uma boa alternativa de substituição aos adesivos convencionais, pelo menos para uso em que o cisalhamento é exigido, sobretudo devido ao seu baixo custo.

\section{CONCLUSÕES}

- A resistência na linha de cola para todas as espécies e a porcentagem de falha na madeira foi maior quando aplicado o adesivo PVAc em relação ao adesivo PUR, embora algumas espécies não diferiram no ensaio de CLC.

- $\quad$. lecointei, E. uncinatum, M. elatum, H. incanus, D. odorata e A. decandra obtiveram eficiência superior a $90 \%$ para o adesivo PVAc

- Para o adesivo PUR as espécies E. uncinatum, M. elatum, H. incanus, D. odorata e A. decandra alcançaram a eficiência maior que $90 \%$. 
- Indica-se P. lecointei, E. uncinatum, M. elatum, H. incanus, D. odorata e A. decandra para a utilização na manufatura de PEM que demandem a aplicação de adesivos, respeitandose a utilização do PVAc para uso interno e o PUR para uso externo.

\section{AGRADECIMENTOS}

O autor expressa seu agradecimento à Coordenação de Aperfeiçoamento de Pessoal de Nível Superior (CAPES), pelo apoio financeiro.

\section{REFERÊNCIAS}

Albuquerque, C. E. C., \& Latorraca, J. V. (2000). Influência das características anatômicas da madeira na penetração e adesão de adesivos. Floresta e Ambiente, 7(1), 158-166.

American Society for Testing and Materials - ASTM. (1994). ASTM D143-94: Standard methods of testing small clear specimens of timber (pp. 24-65). West Conshohocken: ASTM International.

American Society for Testing and Materials - ASTM. (2008). ASTM D905-08: Standard test method for strength properties of adhesive bonds in shear by compression loading. West Conshohocken: ASTM International.

American Society for Testing and Materials - ASTM. (2014). ASTM D2395-14: Standard Test Method for Density and Specific Gravity (Relative Density) of Wood and Wood-Based Materials. Annual Book of ASTM Standards. West Conshohocken: ASTM International.

Bianche, J. J., Teixeira, A. P. M., Ladeira, J. P. S., Carneiro, A. C. O., Castro, R. V. O., \& Della Lucia, R. M. (2017). Cisalhamento na Linha de Cola de Eucalyptus sp. Colado com Diferentes Adesivos e Diferentes Gramaturas. Floresta e Ambiente, 24(0), 1-9. http://dx.doi.org/10.1590/2179-8087.077114.

Biazzon, J. C., Araujo, V. A., Alves, P. R. G., Morales, E. A. M., Gonçalves, M. T. T., \& Valarelli, I. D. D. (2019). Resistência mecânica à adesão em superfícies de madeira de pinus aplainadas e unidas por adesivos PVAc. Matéria (Rio de Janeiro), 24(1), e-12323. http://dx.doi.org/10.1590/s1517707620190001.0606.

Faria, D. L., Mendes, L. M., \& Guimarães Júnior, J. B. (2020). Estudo do comportamento mecânico de madeira laminada colada de Hevea brasiliensis produzida com adesivo poliuretano. Scientia Forestalis, 48(126), 1-11. http://dx.doi.org/10.18671/scifor.v48n126.11.

Ferreira, B. S., Campos, C. I., Silva, M. S., \& Valarelli, I. D. (2012). Cisalhamento na linha de cola de compensados de Eucalyptus sp. e adesivo PVA. Floresta e Ambiente, 19(2), 141-146. http://dx.doi.org/10.4322/floram.2012.016.

He, Q., Zhan, T., Zhang, H., Ju, Z., Hong, L., Brosse, N., \& Lu, X. (2019). Variation of surface and bonding properties among four wood species induced by a high voltage electrostatic field (HVEF). Holzforschung, 73(10), 957-965. http://dx.doi.org/10.1515/hf-2018-0190.

Iwakiri, S. (2005). Painéis de madeira reconstituída. Curitiba, PR: FUPEF.

Iwakiri, S., Cunha, A. B., Alburquerque, C. E. C., Gorniak, E., \& Mendes, L. M. (2000). Utilização de extensores alternativos na produção de compensado multilaminados. Ciência Florestal, 10(1), 77-83. http://dx.doi.org/10.5902/19805098396.

Jakes, J. E., Stone, D. S., \& Frihart, C. R. (2007). Nanoindentation size effects in wood. In Proceedings 30th annual meeting of the adhesion society (pp. 15-17). Miscellaneous Publication.

Lopes, M. C., Muniz, G. I. B., Matos, J. L. M., Tanobe, V. O. A., Chinasso, C. A. F., \& Rosso, S. (2013). Resistência da linha de cola de painéis de Pinus taeda colados lateralmente com diferentes adesivos. Cerne, 19(4), 613-619. http://dx.doi.org/10.1590/S0104-77602013000400011.

Mendoza, Z. M. S. H., Borges, P. H. D. M., Ribeiro, A. S., \& Fernandes, F. B. (2017). Aspectos gerais sobre adesivos para madeira. Multitemas, 22(51), 49-68.

Nascimento, A. M., Garcia, R. A., \& Della Lucia, R. M. (2013). Qualidade de adesão de juntas coladas de diferentes espécies comerciais de madeira. Cerne, 19(4), 593-601. http://dx.doi.org/10.1590/S010477602013000400009.

Oliveira, M. P., \& Seraphim, L. F. (2015). Resistência ao cisalhamento das juntas adesivas produzidas com três diferentes espécies de madeiras e adesivos vinílicos para aplicações não estruturais. Revista Árvore, 39(2), 375-384. http://dx.doi.org/10.1590/0100-67622015000200017.

Roffael, E. (2016). Significance of wood extractives for wood bonding. Applied Microbiology and Biotechnology, 100(4), 1589-1596. PMid:26685670. http://dx.doi.org/10.1007/s00253-015-7207-8. 
Santiago, S. B., Gonçalves, F. G., Lelis, R. C. C., Segundinho, P. G. A., Paes, J. B., \& Arantes, M. D. C. (2018). Eucalypts wood glue with natural adhesives. Revista Materia, 23(3), e-12151.

Santos, C. M. T., \& Del Menezzi, C. H. S. (2010). Efeito da gramatura sobre a resistência ao cisalhamento da linha de cola de duas madeiras tropicais: seru (Allantoma lineata) e Marupá (Simarouba amara). Floresta, 40(2), 345-354. http://dx.doi.org/10.5380/rf.v40i2.17830.

Segundinho, P. G. A., Gonçalves, F. G., Gava, G. C., Tinti, V. P., Alves, S. D., \& Regazzi, A. J. (2017). Eficiência da colagem de madeira tratada de Eucalyptus cloeziana F. Muell para produção de madeira laminada colada (MLC). Revista Materia, 22(2), 1-13.

Souza, E. S. C., Hurtado De Mendoza, Z. M. S., Borges, P. H. M., \& França, G. S. (2020). Propriedades químicas de madeiras nativas comerciais. Multitemas, 25(59), 113-126. http://dx.doi.org/10.20435/multi.v21i59.2803.

Stragliotto, M. C., Pereira, B. L. C., \& Oliveira, A. C. Indústrias madeireiras e rendimento em madeira serrada na Amazônia Brasileira. In: R. J. Oliveira (Ed.). Engenharia florestal: desafios, limites e potencialidade (pp. 499-518). Guarujá: Científica Digital, 2020. http://dx.doi.org/10.37885/200801030.

Varanda, L. D., Yamaji, F. M., Santos, L. R. O., Silva, D. A. L., Christoforo, A. L., \& Lahr, F. A. R. (2018). Physical performance of particleboards using castor oil-based adhesive. Revista Brasileira de Engenharia Agrícola e Ambiental, 22(10), 707-712. http://dx.doi.org/10.1590/18071929/agriambi.v22n10p707-712.

Contribuição dos Autores: TSP: Curadoria de Dados, Metodologia, Administração do Projeto, Software, Escrita Primeira Redação, Escrita - Revisão e Edição; PW: Curadoria de Dados, Supervisão, Metodologia; HRC: Curadoria de Dados, Metodologia; LR: Curadoria de Dados, Metodologia; CHSDM: Supervisão, Análise Formal. 